\title{
Interference of Impulses, Scattered and Radiated by Bodies of Spheroidal Form
}

\author{
Alexander Kleshchev \\ Saint - Petersburg State Navy Technical University, 190008, Saint - Petersburg, Lotsmanskaya st., 3, Russia \\ *Corresponding Author: alexalex-2@yandex.ru
}

Copyright $@ 2013$ Horizon Research Publishing All rights reserved.

\begin{abstract}
With the help of the Fourier transform and characteristics of the scattering of the stationary (continuous) sound signal are calculated the interference of the impulses, scattered and radiated by bodies of the prolate spheroidal form (ideal and elastic), with the harmonic and the frequency-modulated filling.
\end{abstract}

Keywords Interference, Spheroidal Body, Elastic Body, Boundary Conditions

\section{Introduction}

For ideal prolate spheroids, imitating the school, are calculated the interference of scatte-

red impulses, but for elastic spheroidal shells - the interference of radiated impulses (with the help of the dynamic theory of the elasticity, characteristics of the scattering of the stationary sound and the reciprocity theorem). By the scattering of sound by bodies of the spheroidal form had devoted works $[1-8]$, by experiments $-[9,10]$.

\section{Interference of Impulses, Scattered by the School}

The interference of impulses with the harmonic filling, reflected by the school, are learnt

In [11]. In this paper we will study signals in the form of impulses with the harmonic or frequen-cy - modulated filling. At first we consider the school from three fishes, approximated by three soft prolate spheroids (see Fig. 1), illuminated impulses with the harmonic or frequency - modulated filling. The distance between scatterers are chosen with the help of calculations, fulfilled in [12]. In the process of calculations are found time responses and moduluses of spect-rums of scattered impulses of separate scatterers and the summarized reflected impulse. The angle of the illumination $\theta_{0}$ was taken three values: $30^{\circ}, 60^{\circ}, 90^{\circ}$. For the impulse of the illumination $\Psi_{i}(t)$ with the harmonic filling of the frequency $\omega_{0}=2 \pi v_{0}$ his spectrum $S_{0}(2 \pi v)$ has the appearance [13]:

$$
S_{0}(2 \pi v)=\frac{i v_{0}}{\pi\left(v_{0}^{2}-v^{2}\right)}(-1)^{n} \sin \left(\pi n v / v_{0}\right)
$$

where $T$ - the period of oscillations with the frequency $v_{0}, T=1 / v_{0} ; n-$ the number of periods in the impulse; $v$ -the frequency. The impulse of the illumination $\Psi_{i}(t)$ and the modulus of his spectrum $S_{0}(v)$ are presented at Fig.2. 
a)

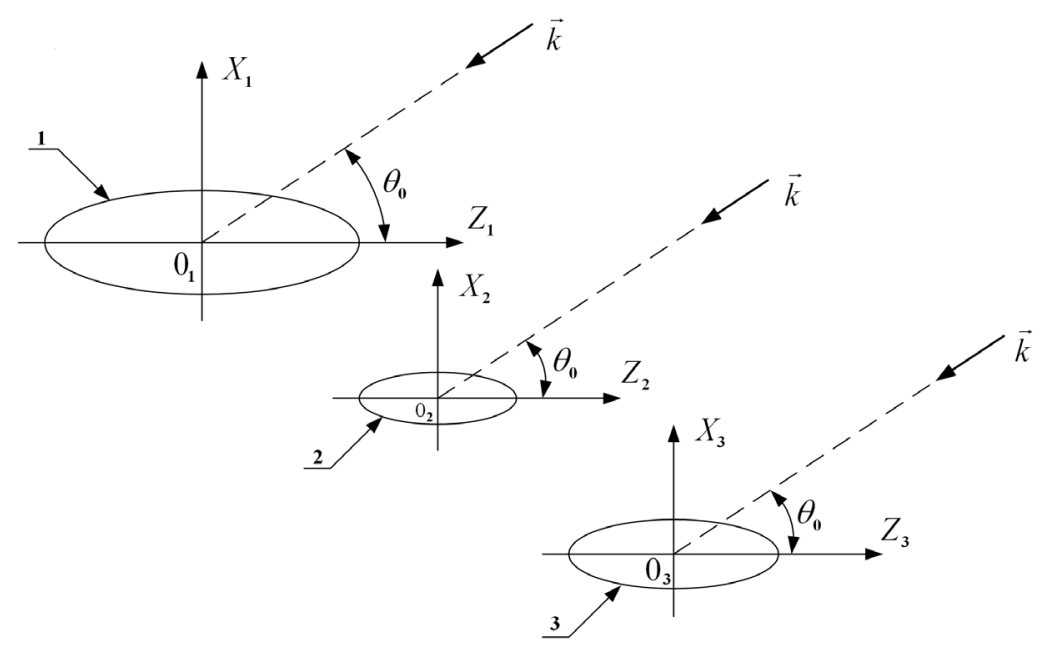

б)

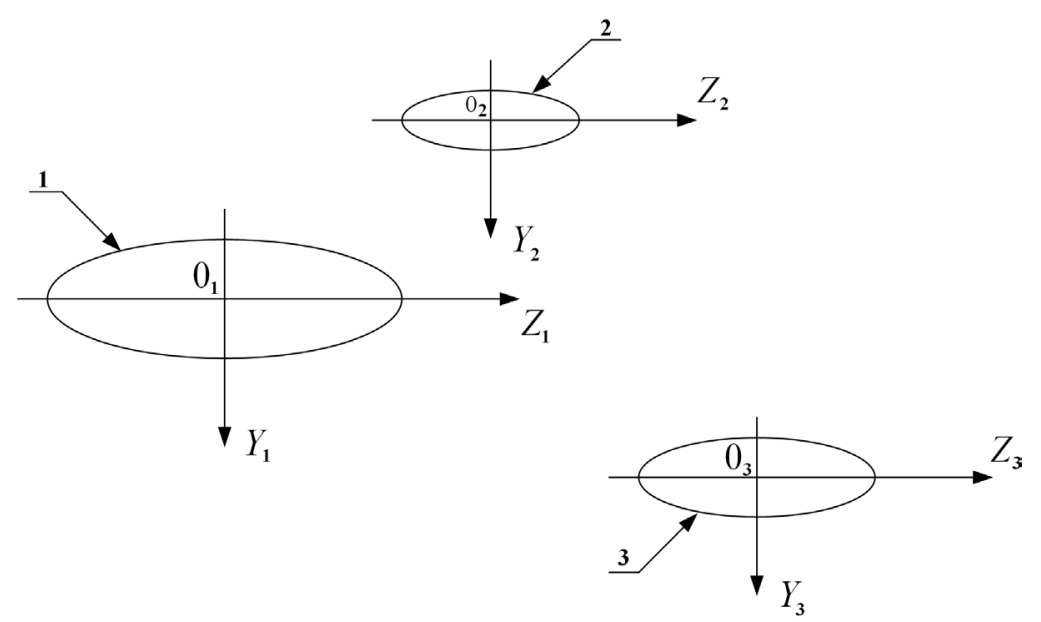

Figure 1. The plan of the school from three fishes: a) the side view; b) the view from above.

a)

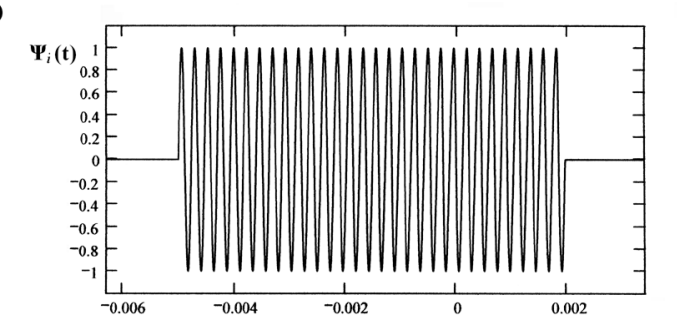

б)

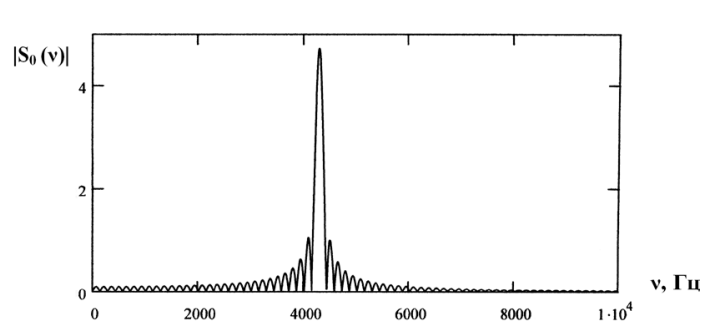

Figure. 2. The impulse of the illumination or the excitation with the harmonic filling $\Psi_{i}(t)$ (a) and the normalized modulus of his spectrum $\left|S_{0}(v)\right|$ (b)

In the frequency - modulated impulse $\Psi_{0}(t)$ the frequency is changed at the linear depen-dence:

$$
\omega=\omega_{0}+a t
$$

where: $a=1,626 \cdot 10^{6}$

The spectrum $S_{01}(2 \pi v)$ of the frequency - modulated impulse is determined at the formula:

$$
S_{01}(2 \pi v)=\int_{-n T / 2}^{+n T / 2} e^{-i 2 \pi v t} \cdot \sin \left[\left(2 \pi v_{0}+a t\right) \cdot t\right] d t
$$

The impulse of the illumination with the frequency - modulated filling $\Psi_{0}(t)$ and the modulus his spectrum $S_{0}(v)$ are represented at Fig. 3. 
a)

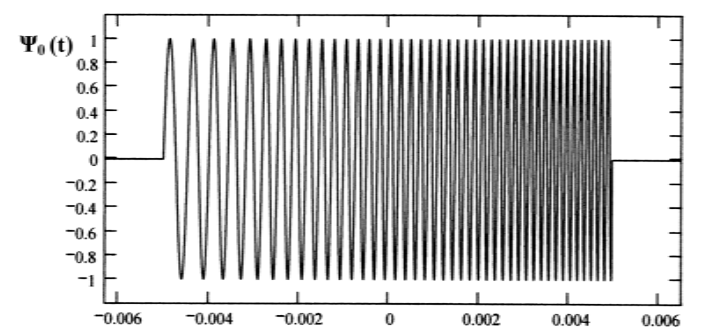

b)

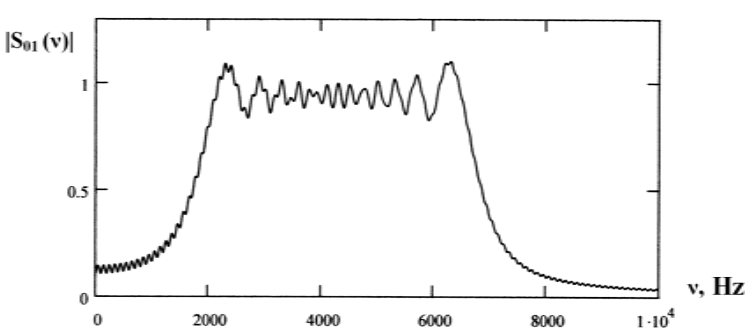

Figure 3. The impulse of the illumination or the excitation with the frequency-modulated filling $\Psi_{0}(t)$ (a) and the normalized modulus of his spectrum $\left|S_{01}(v)\right|($ b).

The spectrum of the scattered signal at the set direction $\theta, \varphi-S_{s}(2 \pi v ; \theta, \varphi)$ and the spectrum of the radiated signal at the direction $\theta, \varphi-S_{q}(2 \pi \nu ; \theta, \varphi)$ were found as the product of the spectrum $S_{0}(2 \pi \nu)$ or $S_{01}(2 \pi \nu)$ incidenting (the problem of the diffraction) or exciting (the pro-blem of the radiation) impulses on the frequency response of the scattering $D_{s}(2 \pi \nu ; \theta, \varphi)$ or the frequency response of the radiation $D_{q}(2 \pi \nu ; \theta, \varphi)$ according. With the help $S_{s}(2 \pi v ; \theta, \varphi)$ and $S_{q}(2 \pi v ; \theta, \varphi)$ are found imsges of scattered [ $\left.\Psi_{s}(t)\right]$ and radiated [ $\Psi_{q}(t)$ ] impulses:

$$
\begin{gathered}
\Psi_{s}\left(t^{\prime}\right)=\pi^{-1} \operatorname{Re} \int_{0}^{\infty} S_{s}(2 \pi v ; \theta, \varphi) \cdot e^{+i 2 \pi v t^{\prime}} \cdot d(2 \pi v) \\
\Psi_{q}\left(t^{\prime}\right)=\pi^{-1} \operatorname{Re} \int_{0}^{\infty} S_{q}(2 \pi v ; \theta, \varphi) \cdot e^{+i 2 \pi v t^{\prime}} \cdot d(2 \pi v)
\end{gathered}
$$

For the determination of spectrums $S_{s}(2 \pi \nu ; \theta, \varphi)$ and $S_{q}(2 \pi \nu ; \theta, \varphi)$ are used characte-ristics of the scattering or the radiation of the sound by spheroidal bodies for the harmonic signal [14]. On the Fig. 4 are represented scattered impulses for physical models of three fishes, finding at distances $r_{1}, r_{2}$ and $r_{3}$, at that $r_{2}-r_{1}<<r_{1}$ and $r_{3}-r_{1}<<r_{1}$ (Fig. 4), the first scatterer in 1,5 times as large of the second scatterer and in 1,2 times as large of the third scatterer. The time $t_{0}$ corresponds to the time of the transit of the scattered signal from the first body until the point of the observation, that is $t_{0}=r_{1} / c$, where $c$ - the velocity of the sound in the liquid. From the interference of third scattered impulses is arisen the signal $\Psi_{s_{\Sigma}}(t)$ (Fig. 5-a), at Fig.5-b repre-sents the normalized modulus of his spectrum $\left|S_{s_{\Sigma}}(v)\right|$, at Fig. 4 and 5 the angle of the illumine-tion $\theta_{0}$ is equal $90^{\circ}$. 
a)

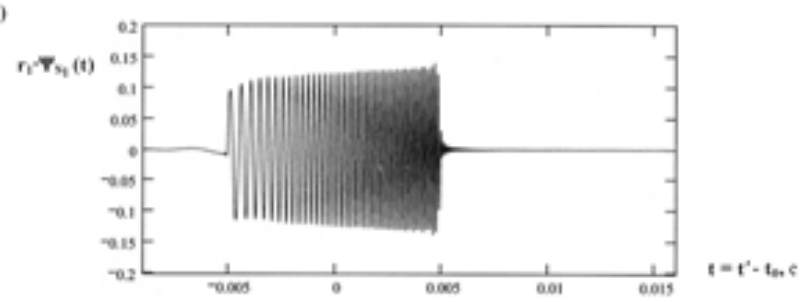

6)
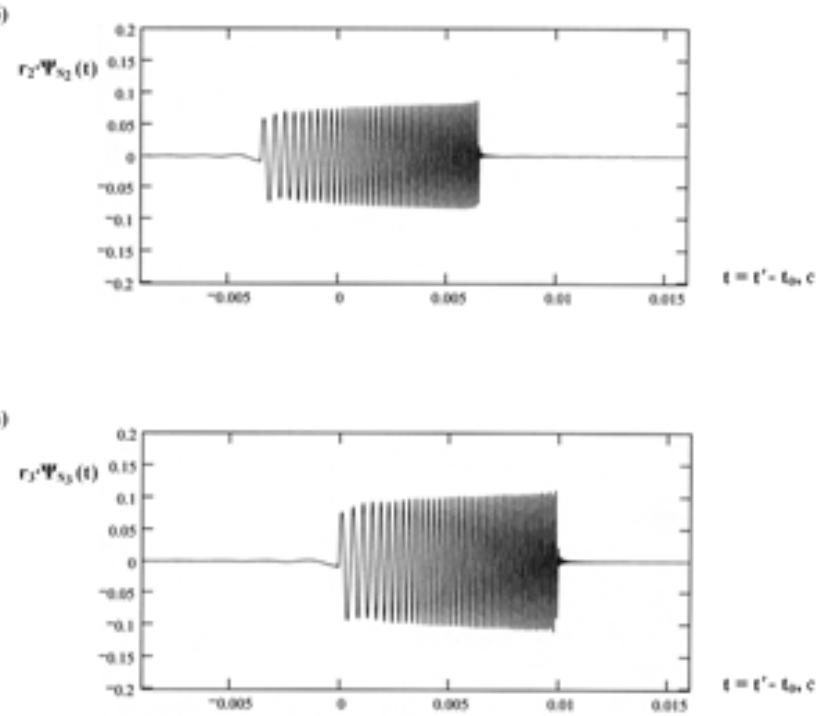

Figure 4. Scattered impulses with frequency - modulated filling for three bodies of the spheroidal forrn of different sizes; $\theta_{0}=90^{\circ}$.

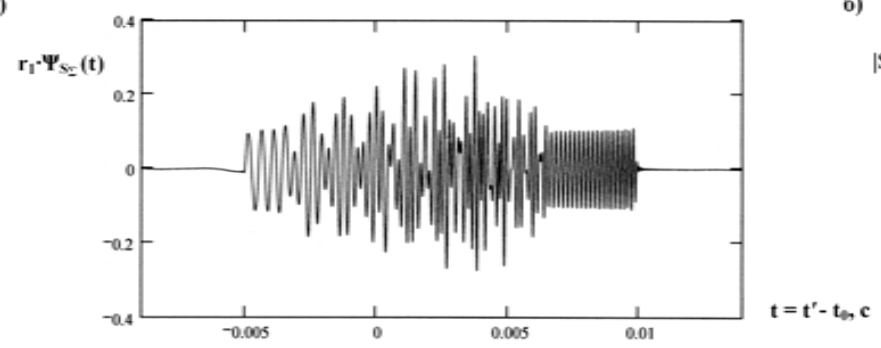

б)

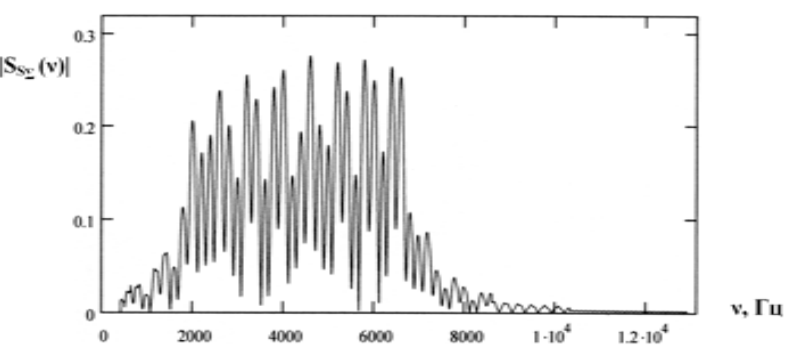

Figure 5. The summarized reflected impulse with frequency - modulated filling $\Psi_{S_{\Sigma}}(t)$ (a) and the normalized modulus of his spectrum $\left|S_{s_{\Sigma}}(v)\right|$ (b); $\theta_{0}=90^{\circ}$

The same calculation by the angle of the illumination $\theta_{0}=90^{\circ}$ was fulfiled for impulses with the harmonic filling (see Fig. 6 and 7). 
a)

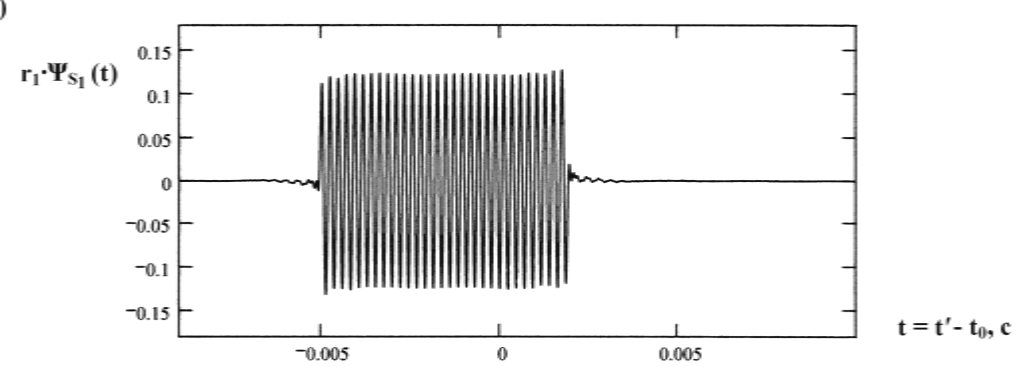

б)



Figure 6. Reflected impulses with the harmonic filling for three bodies of spheroidal form of different sizes, $\theta_{0}=90^{\circ}$.

a)



б)

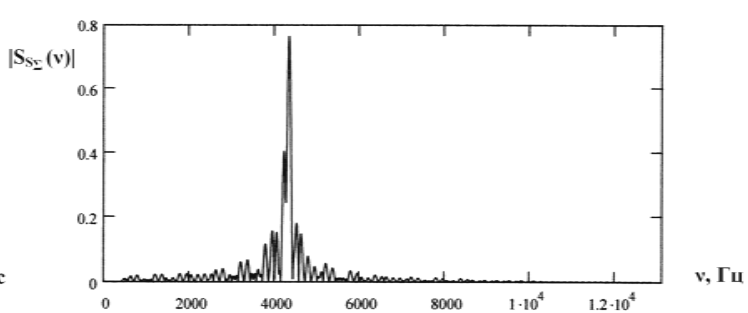

Figure 7. The summarized reflected impulse with thr harmonic filling $\Psi_{S_{\Sigma}}(t)$ (a) and the normalized modulus of his spectrum $\left|S_{S_{\Sigma}}(v)\right|$ (b).

The comparison of Fig. 5 and 7 demonstrates, what impulses with harmonic filling more stable to the interference, that impulses with the frequency - modulated filling.

\section{The Interference of Radiated Impulses}

At Fig. 8 is represented the plan of the disposition of two elastic prolate spheroidal shells, exciting in points $A_{1}$ and $A_{2}$ by impulses with harmonic (Fig. 2) and frequency - modulated (Fig. 3) fillings, the disposition of points $A_{1}$ and $A_{2}$ corresponds by the spheroidal angle $\theta_{0}=0^{\circ}$ (the axis - symmetrical problem)

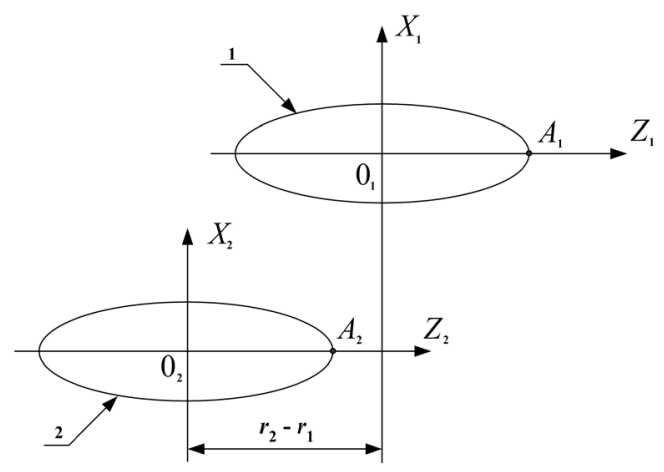

Figure 8. The plan of two spheroidal shell, exciting in points $A_{1}$ and $A_{2}$ by pulsed signals with the frequency - modulated filling $\left(A_{1}\right)$ and the harmonic filling $\left(A_{2}\right) ; \theta_{0}=0^{\circ}$ 
This problem was decided in [14 - 16], we take advantage of this solution. Let us consider a scatterer in the form of an isotropic spheroidal shell, illuminating along axis of the rotation of the shell (the axis - symmetrical problem). All the potentials, including the plane wave potential $\Phi_{0}$, the scattered wave potential $\Phi_{1}$, the scalar shell potential $\Phi_{2}$, the component $A_{\varphi}$ of the vector $\vec{A}$ potential and the potential $\Phi_{3}$ of the gas filling the shell, can be expanded in spheroidal wave functions [14-16]:

$$
\begin{gathered}
\Phi_{0}=2 \sum_{n=0}^{\infty} i^{-n} \bar{S}_{0, n}(C, 1) \bar{S}_{0, n}(C, \eta) R_{0, n}^{(1)}(C, \xi) \\
\Phi_{1}=2 \sum_{n=0}^{\infty} B_{n} \bar{S}_{0, n}(C, \eta) R_{0, n}^{(3)}(C, \xi) \\
\Phi_{2}=2 \sum_{n=0}^{\infty}\left[C_{n} R_{0, n}^{(1)}\left(C_{l}, \xi\right)+D_{n} R_{0, n}^{(2)}\left(C_{l}, \xi\right)\right] \bar{S}_{0, n}\left(C_{l}, \eta\right) ; \\
A_{\varphi}=4 \sum_{n=1}^{\infty}\left[F_{n} R_{1, n}^{(1)}\left(C_{t}, \xi\right)+G_{n} R_{1, n}^{(2)}\left(C_{t}, \xi\right)\right] \bar{S}_{1, n}\left(C_{t}, \eta\right) \\
\Phi_{3}=2 \sum_{n=0}^{\infty} E_{n} R_{0, n}^{(1)}\left(C_{1}, \xi\right) \bar{S}_{0, n}\left(C_{1}, \eta\right)
\end{gathered}
$$

where: $\bar{S}_{m, n}\left(C_{1}, \eta\right)$ - the angular spheroidal function; $R_{m, n}^{(1)}\left(C_{1}, \xi\right), R_{m, n}^{(2)}\left(C_{1}, \xi\right)$ and $R_{m, n}^{(3)}\left(C_{1}, \xi\right)$ - radial spheroidal functions of first, second and third genders corresponding;

$C_{l}=k_{l} h_{0}, k_{l}-$ the wavenumber of the longitudinal elastic wave, $h_{0}-$ semi - focus distance; $C_{t}=k_{t} h_{0}, k_{t}-$ the wavenumber of the transverse elastic wave; $C=k h_{0}, k-$ the wavenumber of the sound wave in the fluid; $C_{1}=k_{1} h_{0}, k_{1}$ - the wavenumber of the sound wave in the gas filling the shell; $B_{n}, C_{n}, D_{n}, F_{n}, G_{n}, E_{n}$ are unknown expansion coefficients. The expansion coefficients are de4termined from the physical boundary conditions preset at the two surfaces of the shell $\left(\xi_{0}\right.$ and $\left.\xi_{1}\right)$ :

1) the continuity of the normal displacement component at both of the boundaries, $\xi_{0}$ and $\xi_{1}$;

2) the identity between the normal stress in the elastic shell and the sound pressure in the liguid $\left(\xi_{0}\right)$ or in the gas $\left(\xi_{1}\right)$;

3) the absence of tangential stress at both of the shell boundaries, $\xi_{0}$ and $\xi_{1}$.

The corresponding expressions for the boundary conditions have in the form [14 - 16]:

$$
\begin{array}{r}
-\left(h_{\xi}\right)^{-1}(\partial / \partial \xi)\left(\Phi_{0}+\Phi_{1}\right)=-\left(h_{\xi}\right)^{-1}\left(\partial \Phi_{2} / \partial \xi\right)+\left(h_{\eta} h_{\varphi}\right)^{-1}\left[(\partial / \partial \eta)\left(h_{\varphi} A_{\varphi}\right)\right] \text { by } \xi=\xi_{0} ;(11) \\
-\left(h_{\xi}\right)^{-1}\left(\partial \Phi_{3} / \partial \xi\right)=-\left(h_{\xi}\right)^{-1}\left(\partial \Phi_{2} / \partial \xi\right)+\left(h_{\eta} h_{\varphi}\right)^{-1}\left[(\partial / \partial \eta)\left(h_{\varphi} A_{\varphi}\right)\right] \text { by } \xi=\xi_{1} ;(12) \\
\Lambda_{0} k^{2}\left(\Phi_{0}+\Phi_{1}\right)=\Lambda_{1} k_{l}^{2} \Phi_{2}+2 \mu_{1}\left\{-\left(h_{\xi} h_{\eta}\right)^{-1}\left(\partial h_{\xi} / \partial \eta\right)\left[\left(h_{\eta}\right)^{-1}\left(\partial \Phi_{2} / \partial \eta\right)+\left(h_{\xi} h_{\varphi}\right)^{-1} \partial\left(h_{\varphi} A_{\varphi}\right) / \partial \xi\right]+\right. \\
\left.+\left(h_{\xi}\right)^{-1}(\partial / \partial \xi)\left[-\left(h_{\xi}\right)^{-1}\left(\partial \Phi_{2} / \partial \xi\right)+\left(h_{\eta} h_{\varphi}\right)^{-1} \partial\left(h_{\varphi} A_{\varphi}\right) / \partial \eta\right]\right\} \text { by } \xi=\xi_{0} ;(13) \\
\Lambda_{2} k_{1}^{2} \Phi_{3}=\Lambda_{1} k_{l}^{2} \Phi_{2}+2 \mu_{1}\left\{-\left(h_{\xi} h_{\eta}\right)^{-1}\left(\partial h_{\xi} / \partial \eta\right)\left[\left(h_{\eta}\right)^{-1}\left(\partial \Phi_{2} / \partial \eta\right)+\left(h_{\xi} h_{\varphi}\right)^{-1} \partial\left(h_{\varphi} A_{\varphi}\right) / \partial \xi\right]+\right. \\
\left.+\left(h_{\xi}\right)^{-1}(\partial / \partial \xi)\left[-\left(h_{\xi}\right)^{-1}\left(\partial \Phi_{2} / \partial \xi\right)+\left(h_{\eta} h_{\varphi}\right)^{-1} \partial\left(h_{\varphi} A_{\varphi}\right) / \partial \eta\right]\right\} \text { by } \xi=\xi_{1} ;(14) \\
-\left[\left(h_{\xi} h_{\eta}\right)^{-1}\left(\partial h_{\eta} / \partial \xi\right)-\left(h_{\xi}\right)^{-1}(\partial / \partial \xi)\right]\left[\left(h_{\eta}\right)^{-1}\left(\partial \Phi_{2} / \partial \eta\right)+\left(h_{\xi} h_{\varphi}\right)^{-1} \partial\left(h_{\varphi} A_{\varphi}\right) / \partial \xi\right]+\left[\left(h_{\xi} h_{\eta}\right)^{-1}\left(\partial h_{\xi} / \partial \eta\right)-\right.
\end{array}
$$




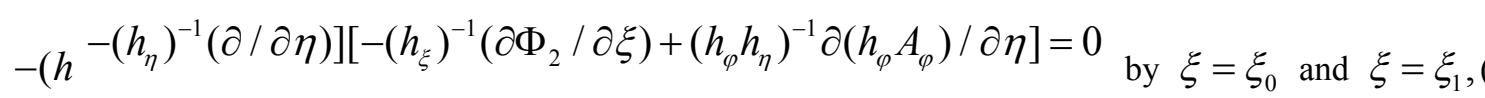

where $h_{\xi}=h_{0}\left(\xi^{2}-\eta^{2}\right)^{1 / 2}\left(\xi^{2}-1\right)^{-1 / 2} ; h_{\eta}=h_{0}\left(\xi^{2}-\eta^{2}\right)^{1 / 2}\left(1-\eta^{2}\right)^{-1 / 2} ; h_{\varphi}=h_{0}\left[\left(1-\eta^{2}\right)\left(\xi^{2}-1\right)\right]^{1 / 2}$;

$\Lambda_{0}$ is the bulk coefficient of the liquid; $\Lambda_{1}$ and $\mu_{1}$ are Lame constans of the spheroidal shell;

$\Lambda_{2}$ is the bulk compression coefficient of the gas filling the shell.

The substitution of series (6) - (10) in boundary conditions (11) - (15) yields an infinite system of equations for determining the desired coefficients. The infinite system is solved by the truncation method. The number of retained terms of expansions (6) - (10) is the greater, the greater the wave size for the given potential.

At the Fig. 9 are represented responses of shells an exciting impulses with the frequency - modulated filling (a) and the harmonic filling (b), but at Fig. 10 is presented summarized impulse

$\Psi_{q_{\Sigma}}(t)$ (a) and the normalized modulus of his spectrum $\left|S_{q_{\Sigma}}(v)\right|(\mathrm{b})$.

The presence in the summarized impulse of the impulse with the harmonic filling smoothes over the negative action of the interference at the summarized impulse.

a)

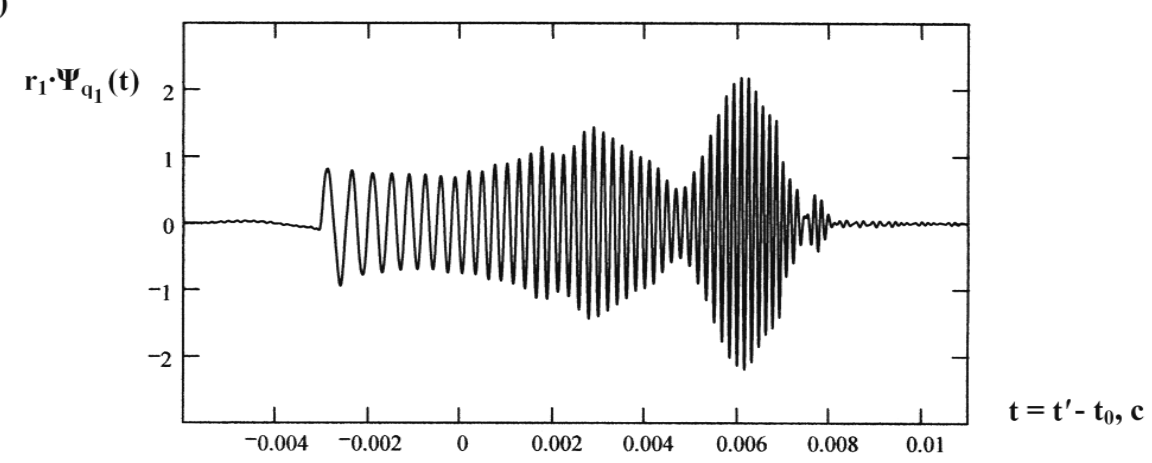

б)

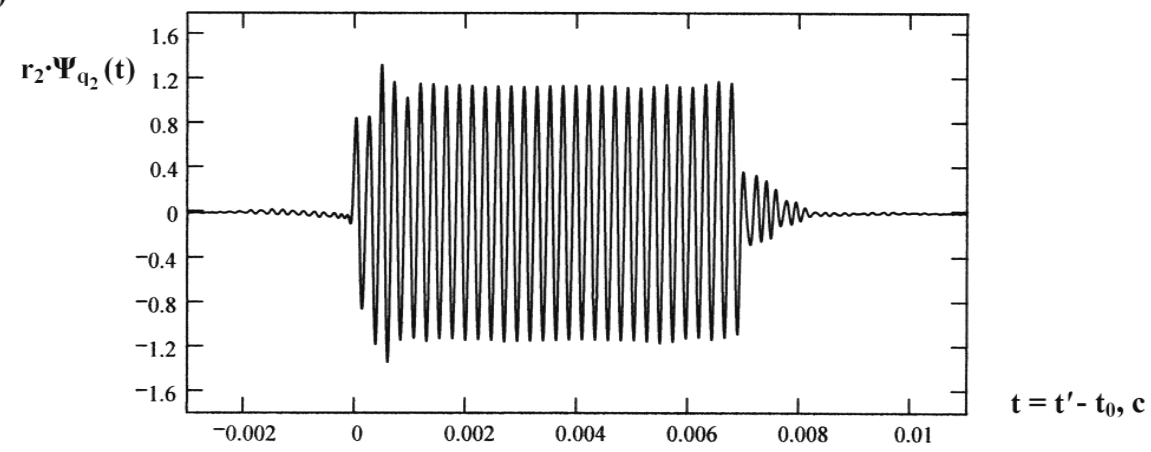

Figure 9. Responses of shells at impulses with the frequency - modulated filling (a) and the harmonic filling (b);

a)

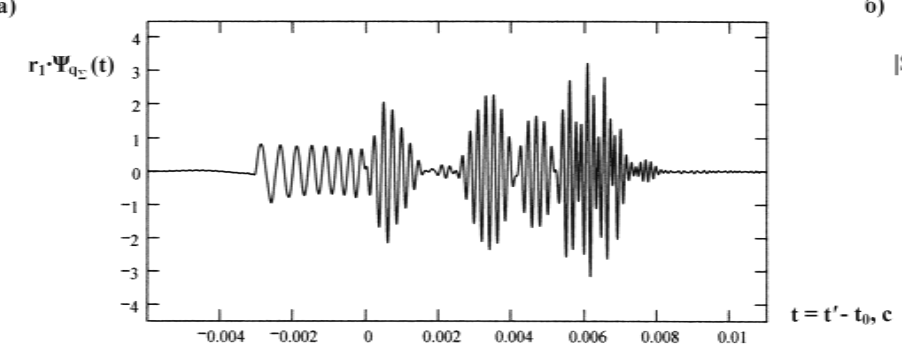

б)

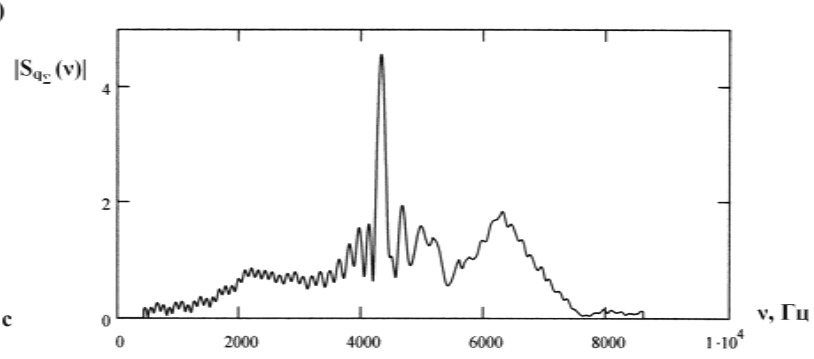

Figure 10. The summarized impulse of the response $\Psi_{q_{\Sigma}}(t)$ in the point of the observation and the normalized modulus of his spectrum $\left|S_{q_{\Sigma}}(v)\right|$; $\theta_{0}=0^{\circ}$

The stable character of impulses with harmonic filling can see from the comparison of Fig. 11 and 12 (the frequency - 
modulated filling) with Fig. 13 and 14 (the harmonic filling).

The advantage of the application of impulses with the harmonic filling by the interference appears evidently.

a)



б)

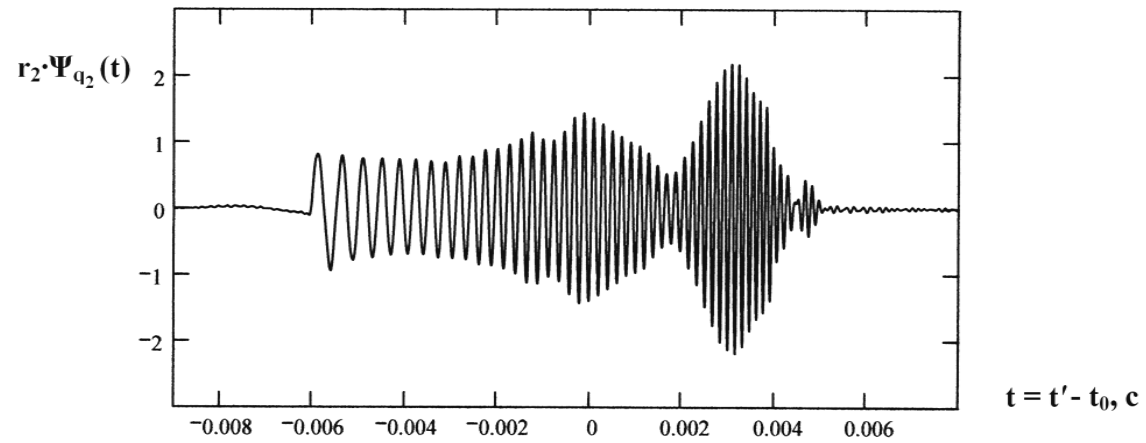

в)



Figure 11. Responses of three spheroidal shells at exciting impulses with the frequency - modu-lated filling





Figure 12. The summarized impulse of the response with the frequency-modulated filling $\Psi_{q_{\Sigma}}(t)$ in the point of the observation and the normalized modulus of his spectrum $\left|S_{q_{\Sigma}}(v)\right| ; \theta_{0}=0^{\circ}$. 
a)

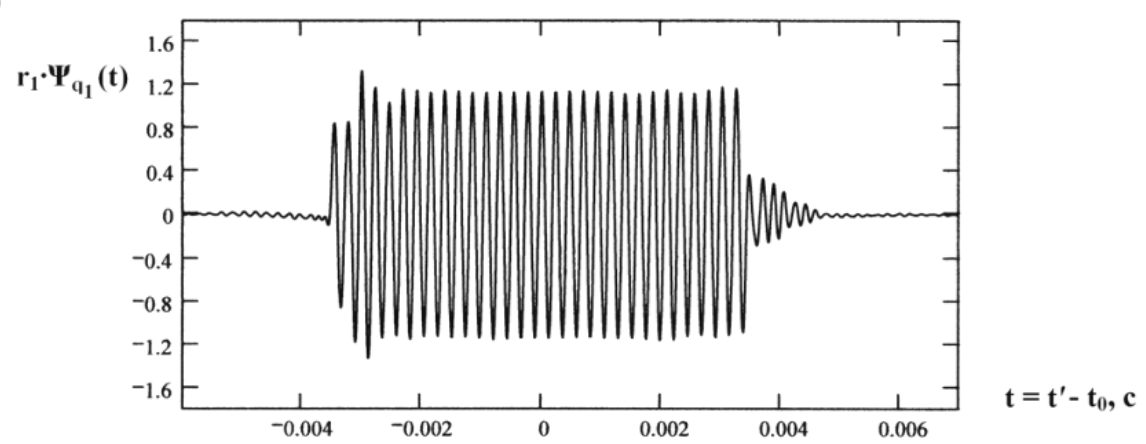

б)



B)

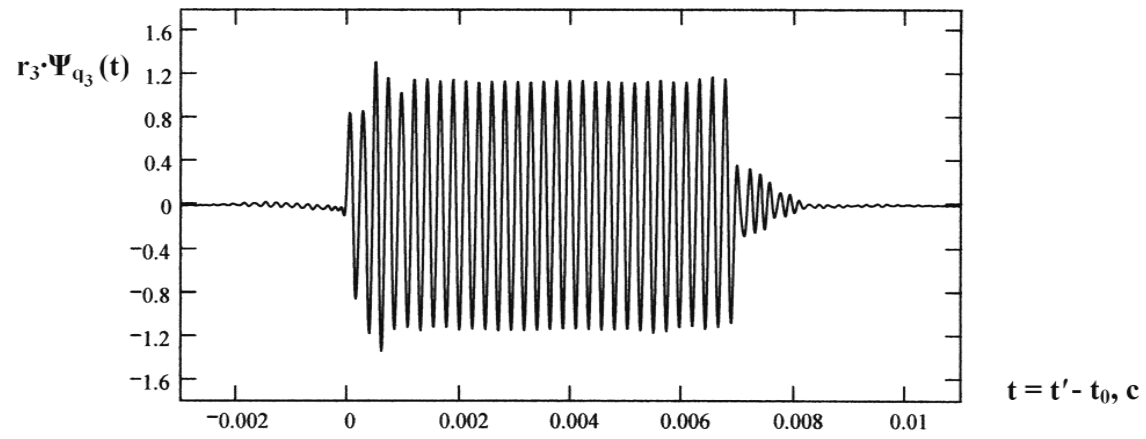

Figure 13. Responses of three spheroidal shells at exciting impulses with the harmonic filling

a)

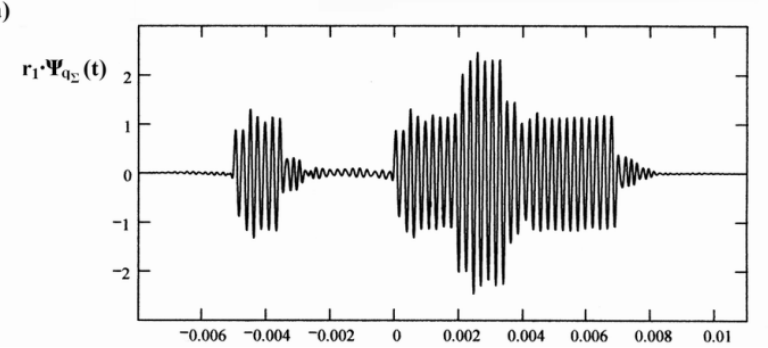

б)

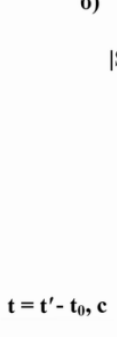

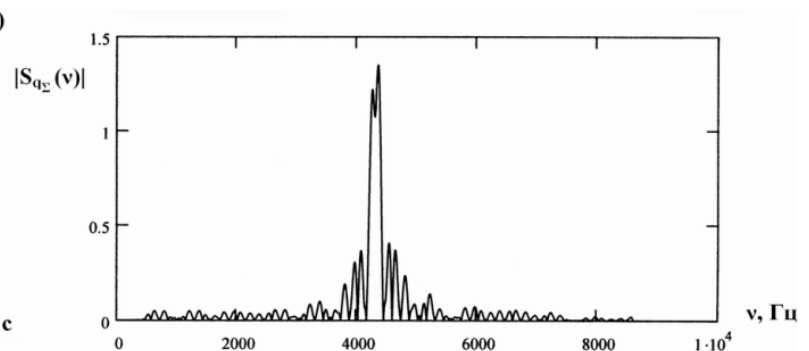

Figure 14. The summarized impulse of thr response with the harmonic filling $\Psi_{q_{\Sigma}}(t)$ in the point of the obser-vation and the normalized modulus of his spectrum $\left|S_{q_{\Sigma}}(v)\right| ; \theta_{0}=0^{\circ}$

\section{Conclusions}

In the paper were calculated scattered and radiated impulses with harmonic and frequency - modulated fillings for ideal and elastic spheroidal bodies. Signals with the harmonic filling appear more stable to the interference. The study of the interference of impulses, scattered and radiated by bodies of the spheroidal form appears by the object of the research in the hydroacoustic. 


\section{Acknowledgments}

The work was supposed as part of research under State Contract no P242 of April 21. 2010, within the Federal Target Program "Human Capital in Science and Education for Innovative Russia, 2009 - 2013".

\section{REFERENCES}

[1] A. A. Kleshchev, L. S. Sheiba. Scattering of sound wave by ideal prolate spheroids. // Acoustical journal. 16, 264 (1970).

[2] A. A. Kleshchev. Scatterer in field of point source. // Acoustical journal. 19, 455 (1973).

[3] A. A. Kleshchev. Scattering of sound by ideal spheroids in limit case of high frequencys. // Acoustical journal. 19, 699 (1973).

[4] A. A. Kleshchev, I. I. Klukin. Compensation of pressure in wave, scattered by ideal spheroid. // Acoustical journal. 20, 252 (1974).

[5] A. A. Kleshchev. Diffraction of sound at bodies with mixed boundary conditions. // Acoustical journal. 20, 632 (1974).

[6] A. A. Kleshchev. Scattering of sound by bodies, finding at boundary of division of mediums. // Acoustical journal.23, 404 (1977).

[7] M. F. Werby, L. H. Green. An Extended Unitry Approach for Acoustical Scattering from Elastic Shells, Immrsed in a Fluid. // J. A. S. A. 74, 625 (1983).

[8] M. F. Werby, L. H. Green. Comparison of Acoustical Scattering from Fluid - Loaded Elastic Shells and Sound - Soft Objects. // J. A. S. A. 76, 1227 (1984).

[9] A. A. Kleshchev. Scattering of Low - Frequency Pulsed Sound Signals from Elastic Cylindrical Shells. // Acoust. Phys. 57, 375 (2011).

[10] A. A. Kleshchev. Measurement of Characteristics of Sound Reflection and Scattering by Elastic Cylindrical Shells in Conditions of Hydroacoustic Basin. // Int. J. of Ph. 1, 5 (2013).

[11] A. A. Kleshchev. Physical model of sound scattering by school, finding at boundary of division of mediums. // Acoustical journal, $50,512(2004)$.

[12] A. A. Kleshchev. Scattering of sound by spheroidal body, finding at boundary of division of mediums. // Acoustical journal, 25, 143(1979).

[13] A. A. Kharcevich. Spektrums and analysis. Moskau, GITTL, 1957 [in Russia].

[14] A. A. Kleshchev. Hydroacoustic Scatterers. First publication, St. Petersburg, Shipbui-lding, 1992; second publication, St. Petersburg, Prima, 2012 [in Russia].

[15] A. A. Kleshchev. Scattering of sound by elastic oblate spheroidal shell. // Acousical journal, 21, 938 (1975).

[16] A. A. Kleshchev, E. I. Kuznetsova. About Low Frequency Resonances of Elastic Sphero-idal Shells, Irradiating and Establishing by Harmonic and Pulse Signals. // I. J. T. M. P. 3, 4 (2013). 